\title{
THE STRONGER FORM OF CAUCHY'S INTEGRAL THEOREM
}

\author{
E. F. BECKENBACH
}

1. Introduction. The so-called weaker and stronger forms ${ }^{1}$ of Cauchy's integral theorem are the following.

Theorem 1. Cauchy's integral theorem (WEAKer Form). If $f(z)$ is holomorphic on the finite simply-connected open domain $D$, and if $C$ is a closed rectifiable curve in $D$, then

$$
\int_{C} f(z) d z=0 \text {. }
$$

Theorem 2. Cauchy's integral theorem (stronger Form). If $f(z)$ is holomorphic on the interior $D$ of a simply closed rectifiable curve $C$, and continuous on $D+C$, then

$$
\int_{C} f(z) d z=0 .
$$

Each of these theorems follows readily when it has been established that there is a sequence of closed curves $\left\{C_{n}\right\}$ in $D$, of uniformly bounded length, converging in the sense of Fréchet to $C$, such that

$$
\int_{C_{n}} f(z) d z=0 \text {. }
$$

In the case of Theorem 2 it appears to be more difficult to establish the existence of the sequence $\left\{C_{n}\right\}$, since the convergence must be from only one side; but the difficulty may be overcome by more or less tedious topological considerations, a program which has been undertaken by a number of authors. ${ }^{2}$ Recent excellent proofs by Reid and Hestenes, loc. cit., appear to be about as simple as a valid elementary proof of this result could be.

The above type of proof applies equally well to yield the corresponding stronger form of Green's lemma, as some authors have

Presented to the Society, December 27, 1942; received by the editors November 4, 1942.

1 This terminology is taken from M. H. A. Newman, Elements of the topology of plane sets of points, Cambridge, England, 1939, pp. 154, 156.

${ }^{2}$ For bibliographical discussions see S. Pollard, On the conditions for Cauchy's theorem, Proc. London Math. Soc. vol. 21 (1923) pp. 456-482; E. Kampe, Zu dem Integralsatz von Cauchy, Math. Zeit. vol. 35 (1932) pp. 539-543; W. T. Reid, Green's lemma and related results, Amer. J. Math. vol. 63 (1941) pp. 563-574; M. R. Hestenes, An analogue of Green's theorem in the calculus of variations, Duke Math. J. vol. 8 (1941) pp. 300-311. 
pointed out, and also to yield other integral results such as the analogous stronger form of Cauchy's integral formula, and so on.

Walsh and Heilbronn ${ }^{3}$ have given brief and simple, but not elementary, proofs of Theorem 2, using results of Walsh and Fejér on polynomial boundary approximations to holomorphic functions. Their methods of proof do not yield the stronger form of Green's lemma.

As an application of Theorem 2, consider Painlevé's theorem in the following form.

PaInlevé's Theorem. If open plane domains $D_{1}$ and $D_{2}$ abut along the rectifiable arc $C$, if $f_{j}(z)$ is holomorphic on $D_{j}$ and continuous on $D_{j}+C, j=1,2$, and if $f_{1}(z)=f_{2}(z)$ on $C$, then $f_{1}(z)$ and $f_{2}(z)$ are analytic continuations of one another across $C$.

The proofs of this theorem use the finite accessibility of points of $C$ from $D_{1}$ and from $D_{2}$, together with the stronger form of Cauchy's integral theorem and sometimes of Cauchy's integral formula; the weaker forms do not suffice.

The following proof of Theorem 2, though not elementary, uses only tools which are usually considered standard. It is brief and simple, and the method has the advantage of being equally applicable to establishing the stronger form of Green's lemma, Cauchy's integral formula, and so on.

2. Lemmas. We shall use the following two lemmas.

Lemma 1. Bieberbach-Csillag theorem. ${ }^{4}$ If $z=g(w)$ is holomorphic on $|w|<1$ and continuous on $|w| \leqq 1$, and if $l(r)$ denotes the length of the image of $|w|=r \leqq 1$ on the $z$-plane, then $l(r)$ is a monotonically non-decreasing function of $r$ for $0 \leqq r \leqq 1$.

PRoof. Fix $r<1$ and let $r<R \leqq 1$. Since the length of a curve is the limit of the lengths of a proper sequence of inscribed polygons, for any positive $\epsilon$ there is a set of values $\theta_{1}, \theta_{2}, \cdots, \theta_{n}, \theta_{n+1}=\theta_{1}$, with $\theta_{1}<\theta_{2}<\cdots<\theta_{n}<\theta_{1}+2 \pi$, such that

$$
p(r) \equiv \sum_{j=1}^{n}\left|g\left(r e^{i \theta j+1}\right)-g\left(r e^{i \theta j}\right)\right|>l(r)-\epsilon .
$$

${ }^{3}$ J. L. Walsh, The Cauchy-Goursat theorem for rectifiable Jordan curves, Proc. Nat. Acad. Sci. U.S.A. vol. 19 (1933) pp. 540-541; H. Heilbronn, $Z u$ dem Integralsatz von Cauchy, Math. Zeit. vol. 37 (1933) pp. 37-38.

${ }^{4}$ L. Bieberbach, Über die konforme Kreisabbildung nahezu kreisförmiger Bereiche, Preuss. Akad. Wiss. Sitzungsber, 1924, pp. 181-188. 
Consider the function $p(w) \equiv \sum_{j=1}^{n}\left|g\left(w e^{i \theta j+1}\right)-g\left(w e^{i \theta j}\right)\right|$; now $p(w)$ is continuous for $|w| \leqq 1$; and since the absolute value of a holomorphic function is subharmonic and the sum of a finite number of subharmonic functions is subharmonic, it follows that $p(w)$ is subharmonic for $|w|<1$. Hence $p(w)$ assumes its maximum value on $|w| \leqq R$ at some point $R e^{i \theta_{0}}$ on $|w|=R$; in particular,

$$
p(r) \leqq p\left(R e^{i \theta 0}\right) \text {. }
$$

Clearly $p\left(R e^{i \theta_{0}}\right)$ is the length of a polygon inscribed in the image of $|w|=R$, so that

$$
p\left(R e^{i \theta 0}\right) \leqq l(R) .
$$

Now (2), (3) and (4) yield $l(r)-\epsilon<l(R)$, whence $l(r) \leqq l(R)$.

Lemma 2. Corollary of Helly-Bray theorem. ${ }^{5}$ In the unit circle $r \leqq 1$, let $g\left(r e^{i \theta}\right)$ and $h\left(r e^{i \theta}\right)$ be continuous complex functions and let $g\left(r e^{i \theta}\right)$, as function of $\theta, 0 \leqq \theta \leqq 2 \pi$, be of limited variation, uniform with respect to $r$. Then

$$
\int_{0}^{2 \pi} h\left(e^{i \theta}\right) d g\left(e^{i \theta}\right)=\lim _{r \rightarrow 1} \int_{0}^{2 \pi} h\left(r e^{i \theta}\right) d g\left(r e^{i \theta}\right) .
$$

Proof. Let $T$ be the least upper bound of the total variations $T_{r}$. Since $h\left(r e^{i \theta}\right)$ is uniformly continuous in the unit circle, for any positive $\epsilon$ there is a positive integer $n$ such that for all $r \leqq 1$,

$$
\left|h\left(r e^{i \theta}\right)-h\left(r e^{i \theta \prime}\right)\right| \leqq \epsilon /(3 T)
$$

provided $\left|\theta-\theta^{\prime}\right| \leqq 2 \pi / n$. Then for all $r \leqq 1$, we have $e^{6}$

$$
\begin{aligned}
\mid \int_{0}^{2 \pi} h\left(r e^{i \theta}\right) d g\left(r e^{i \theta}\right)-\sum_{j=1}^{n} h\left(r e^{2 j \pi i / n}\right)[ & g\left(r e^{2 j \pi i / n}\right) \\
& \left.-g\left(r e^{2(j-1) \pi i / n}\right)\right] \mid \leqq \epsilon / 3 .
\end{aligned}
$$

Also, by the continuity of $g\left(r e^{i \theta}\right)$ and $h\left(r e^{i \theta}\right)$ as functions of $r$, there is an $r_{0}<1$ such that for $r_{0}<r<1$,

$$
\begin{aligned}
\mid \sum_{j=1}^{n} h\left(r e^{2 j \pi i / n}\right)[ & \left.g\left(r e^{2 j \pi i / n}\right)-g\left(r e^{2(j-1) \pi i / n}\right)\right] \\
& -\sum_{j=1}^{n} h\left(e^{2 j \pi i / n}\right)\left[g\left(e^{2 j \pi i / n}\right)-g\left(e^{2(j-1) \pi i / n}\right)\right] \mid<\epsilon / 3 .
\end{aligned}
$$

5 See for instance G. C. Evans, The logarithmic potential, Amer. Math. Soc. Colloquium Publications, vol. 6, 1927, pp. 14-16.

${ }^{6}$ Cf. K. Knopp, Funktionentheorie, vol. 1, Berlin, 1930, p. 44, Hilfssatz 2. 
Now (5), taken once for $r_{0}<r<1$ and again for $r=1$, and (6) yield

$$
\left|\int_{0}^{2 \pi} h\left(e^{i \theta}\right) d g\left(e^{i \theta}\right)-\int_{0}^{2 \pi} h\left(r e^{i \theta}\right) d g\left(r e^{i \theta}\right)\right|<\epsilon,
$$

and the lemma is established.

3. Proof of Theorem 2. By the Riemann mapping theorem, there is a holomorphic function $z=g(w)$ which maps the interior of the unit circle $|w|<1$ on $D$; further, by the Osgood-Carathéodory theorem, $g(w)$ remains continuous on $|w| \leqq 1$ and $z=g(w)$ maps $|w|=1$ topologically on $C$. Let $f(z)=f[g(w)]=h(w)$. Now $g(w)$ and $h(w)$ are continuous on $|w| \leqq 1$ and, by Lemma $1, g\left(r e^{i \theta}\right)$, as function of $\theta$, $0 \leqq \theta \leqq 2 \pi$, is of limited variation, uniform with respect to $r$. Consequently, by Lemma 2,

$$
\begin{aligned}
\int_{C} f(z) d z & =\int_{0}^{2 \pi} f\left[g\left(e^{i \theta}\right)\right] d g\left(e^{i \theta}\right)=\int_{0}^{2 \pi} h\left(e^{i \theta}\right) d g\left(e^{i \theta}\right) \\
& =\lim _{r \rightarrow 1} \int_{0}^{2 \pi} h\left(r e^{i \theta}\right) d g\left(r e^{i \theta}\right)=\lim _{r \rightarrow 1} \int_{C_{r}} f(z) d z,
\end{aligned}
$$

where $C_{r}$ is the image of $|w|=r$ on the $z$-plane. But by Theorem 1 ,

$$
\int_{C_{r}} f(z) d z=0 \text {. }
$$

Now (1) follows from (7) and (8).

The University of TeXas 